\title{
Physical properties of Hickson compact groups and of the loose groups within which they are embedded ${ }^{\star}$
}

\author{
H. Tovmassian ${ }^{1,2}$, M. Plionis ${ }^{3,1}$, and J. P. Torres-Papaqui ${ }^{1}$ \\ 1 Instituto Nacional de Astrofísica Optica y Electrónica, AP 51 y 216, CP 72000, Puebla, Pue., Mexico \\ e-mail: [hrant, papaqui]@inaoep.mx \\ 2 Sternberg Astronomical Institute, Moscow State University, Moscow, Russia \\ 3 Institute of Astronomy \& Astrophysics, National Observatory of Athens, Palaia Penteli, 15236 Athens, Greece \\ e-mail:mplionis@astro.noa.gr
}

Received 21 October 2005 / Accepted 4 May 2006

ABSTRACT

\begin{abstract}
Using new data and an enlarged group sample we verify some of our previously published results and present a number of new facts that suggest that compact groups could be casual concentrations in prolate-like looser groups, and thus the nature of compact and ordinary poor groups is probably the same.

To this end we used the Sloan Digital Sky Survey (SDSS) redshift catalogue to look for galaxies with accordant redshifts in the nearby environment (up to $\sim 2 \mathrm{Mpc}$ ) of 15 Hickson compact groups (HCG). We also used known member redshifts of looser groups in the environment of 7 other HCGs. From this sample of 22 HCGs we find that: (a) HCG's tend to be aligned with the overall galaxy distribution in their $\sim 1 \mathrm{Mpc}$ environment; (b) the well-established orientation effect by which the group velocity dispersion $\sigma_{v}$ correlates with group axial ratio $q$ is present and particularly strong also in the HCG + environment systems; (c) the velocity dispersion $\sigma_{v}$ of the HCG + environment systems as well as of ordinary poor groups, depends only weakly on the group richness, i.e. on the mass; (d) the mean absolute $K$-band magnitude of E/S0 galaxies in HCGs is similar to the corresponding one in ordinary poor groups and is brighter than that of isolated E/SO's, indicating that they were formed by the merging of two galaxies of similar luminosity; (e) the fraction of E/S0 galaxies in these HCGs depends, albeit weakly, on the group's richness and on $\sigma_{v}$; (f) the fraction of AGNs is similar in the HCGs and their close environment, while the fraction of starburst galaxies is significantly higher in the HCGs; (g) the fraction of active galaxies (AGNs and starbursts) is anti-correlated with the velocity dispersion of the HCG + environment systems.

The combination of all the above facts constitutes a picture in which compact groups are condensations within looser prolate-like, elongated systems, and they appear to be compact when their member galaxies, moving in radial orbits along the group elongation, happen to come close to each other (in which case dynamical interactions among these galaxies become even more probable) or when the group is oriented close to the line of sight, so that many of its members are projected over a small solid angle. The probability of either case is small, so the number of CGs should be much smaller than that of ordinary groups, as observed.

Furthermore, the observed fractions of early-type and active galaxies, as well as their correlations with the group velocity dispersion suggests a picture by which nuclear activity and galaxy transformation by merging is instigated by effective gravitational interactions in the low-velocity dispersion groups, which then dynamically evolve via virialization processes to higher velocity dispersion groups, which thus have a higher fraction of early-type galaxies.
\end{abstract}

Key words. galaxies: general - galaxies: clusters: general - cosmology: large-scale structure of Universe

\section{Introduction}

Compact groups (CGs) are environments with high surface galaxy density and relatively low radial velocity dispersion, $\sigma_{v}$. In recent decades several lists of CGs have been compiled (see Hickson 1997; Focardi \& Kelm 2002; Iovino et al. 2003; Lee et al. 2004; de Carvalho et al. 2005), but the most extensively studied sample of CGs is still that of Hickson compact groups (HCGs, Hickson 1982). $N$-body simulations have shown that strong galaxy interactions and merging should occur frequently in such systems, and CGs should eventually evolve to form a single elliptical galaxy on a time scale of a few orbital periods (Barnes 1985, 1989, 1990; Mamon 1987; Bode et al. 1993). For this reason the very existence of CGs has been questioned and debated. To explain the existence of CGs, it has been suggested that field galaxies fall from time to time onto CGs, thus

* Figure 1 is only available in electronic form at http://www.edpsciences.org keeping the number of groups approximately constant (Diaferio et al. 1994, 1995; Governato et al. 1996; Ribeiro et al. 1998). However, observational estimates (Zepf et al. 1991; Moles et al. 1994; Zepf 1993; Mendes de Oliveira \& Hickson 1994; Coziol et al. 2004) - suggest relatively low merger rates.

To explain these observations Coziol et al. (2004) put forward two hypotheses: either the evolution of galaxies accelerates in richer systems or CG's form earlier in massive structures than in lower-mass systems. Solutions to the problem also come (a) from the fact that CGs are small subsystems in larger ordinary (hereafter, loose) groups (LGs) (Rose 1977; Sulentic 1987; Rood \& Williams 1989; West 1989; Mamon 1990; Vennik et al. 1993; Diaferio et al. 1994; Rood \& Struble 1994; Tovmassian \& Chavushyan 2000; Tovmassian 2001; Tovmassian et al. 2001) and (b) from a sufficiently high formation rate, so that they are still abundant enough to be observed at their present numbers despite their short merging times (e.g. Mamon 2000). Ramella et al. (1994) concluded that we see HCGs because they are being 
continually formed in collapsing LGs, as was predicted by some $N$-body simulations of rich groups of galaxies (e.g. Diaferio et al. 1995).

It has been shown that HCGs, Shakhbazian CGs (Shakhbazian 1973; Baier \& Tiersch 1979, and references therein) as well as poor groups have a prolate-like shape (Malykh \& Orlov 1986; Oleak et al. 1995; Plionis et al. 2004). Tovmassian et al. (1999) show that the velocity dispersion of HCGs correlates with their projected shape: the lower the group's projected axial ratio, the smaller its velocity dispersion. They conclude that member galaxies move in radial orbits predominantly along the group's elongation. Furthermore, Tovmassian (2001), Tovmassian et al. (2001) and Tovmassian \& Tiersch (2001) have shown that members of LGs in the environment of CGs are distributed in narrow strips of a couple of hundred kpc width and $\sim 1 \mathrm{Mpc}$ length, while the projected position angle of the LG generally coincides with that of the CG. Using galaxy redshift data in the environments of 6 HCGs from de Carvalho et al. (1994) and Zabludoff \& Mulchaey (1998), Tovmassian \& Chavushyan (2000) show that accordant redshift galaxies in the environments of these HCGs and poor groups obey the dependence even more strongly of the projected shape on the $\sigma_{v}$ of the whole system. It follows that members of LGs should be gravitationally bound with CGs and have a quasi-regular movement predominantly along the elongation of the whole system. Tovmassian \& Chavushyan (2000) and Tovmassian (2002) put forward the idea that CGs are ordinary poor groups in which the brighter members happen to be close to each other during the orbital movement around the centre of gravity, or if the groups are seen end-on and most of its members are projected near to each other on the sky.

In this paper we present indications of a possible common nature for compact and loose groups of galaxies. We show that CGs are condensations in LGs and that the HCG + environment systems obey similar correlations as loose groups.

\section{The sample}

In order to study the dynamics of HCG + environment systems, we analysed a sample of 22 HCGs. Since the projected mean length of poor groups is generally less than $1 \mathrm{Mpc}^{1}$ (e.g. see Plionis et al. 2004), we looked for accordant redshift galaxies up to a distance of $\sim 2 \mathrm{Mpc}$ from the original HCGs. We used mainly the SDSS (Abazajian et al. 2005) to search for galaxies with a redshift separation from the centre of the corresponding HCG of $\delta(c z)=1000 \mathrm{~km} \mathrm{~s}^{-1}$ and within a circle of projected radius $\sim 2 \mathrm{Mpc}$ radius. In the area covered by the SDSS, we find 22 HCGs out of which 15 (\# 7, 25, 35, 43, 45, 49, 50, 56, 58, 60, $66,68,82,88$, and 98) are sampled out to the projected separation limit discussed previously, while the other 7 are located near the SDSS survey limits, and thus are not sampled adequately. Accordant redshift galaxies were found in the environment of 14 of these 15 groups (see Fig. 1). We noted that the magnitude distribution of the accordant redshift galaxies does not differ in most cases from that of the HCG galaxies.

Furthermore, the environment of 6 other HCGs (HCG 40, HCG 63, HCG 64, HCG 67, HCG 87, and HCG 97) have been studied spectroscopically up to sufficiently large projected distances from their centre (de Carvalho et al. 1997; Zabludoff \& Mulchaey 1998). The environment of one more group, HCG 92 (the well-known Stephan Quintet) has been studied in HI emission by Shostak et al. (1984), who detected 5 accordant redshift

\footnotetext{
1 Throughout this paper we use $H_{0}=72 \mathrm{~km} \mathrm{~s}^{-1} \mathrm{Mpc}^{-1}$.
}

galaxies, and by Williams et al. (2002) who detected one more accordant redshift galaxy (last image in Fig. 1). We assume that the discordant redshift galaxy NGC 7320 is not a member of the group.

We found that the velocity dispersions of a few HCG + environment systems are higher than those of loose groups, that generally do not exceed $400 \mathrm{~km} \mathrm{~s}^{-1}$. Unusually high are the $\sigma_{v}$ 's of groups HCG 50 and HCG 82: $546 \mathrm{~km} \mathrm{~s}^{-1}$ and $468 \mathrm{~km} \mathrm{~s}^{-1}$, respectively. Note that using the original four members of HCG 82 we obtain an even higher value: $\sigma_{v} \simeq 620 \mathrm{~km} \mathrm{~s}^{-1}$. This leads us to suspect that they could be the result of projection effects. The group HCG 50 could well be the superposition of two independent groups. The original four galaxies, $a, b, c$, and $e$ (Hickson designations) of this group could compose two groups: one with $\langle c z\rangle=41520 \mathrm{~km} \mathrm{~s}^{-1}$ and $\sigma_{v}=263 \mathrm{~km} \mathrm{~s}^{-1}$ (HCG 50a) and one with $\langle c z\rangle=42450 \mathrm{~km} \mathrm{~s}^{-1}$ and $\sigma_{v}=96 \mathrm{~km} \mathrm{~s}^{-1}$ (HCG 50b). The radial velocities of the two subgroups differ by $\sim 900 \mathrm{~km} \mathrm{~s}^{-1}$, which corresponds to a distance between two subgroups of about $13 \mathrm{Mpc}$, if viewed as a radial separation. We assume that the group HCG 82 consists of 6 accordant redshift galaxies, $b, c$, and the SDSS galaxies 3, 4, 5, and 6 with $\langle c z\rangle=10550 \mathrm{~km} \mathrm{~s}^{-1}$ and $\sigma_{v}=233 \mathrm{~km} \mathrm{~s}^{-1}$. We suppose that two galaxies, $a$ and $d$ with mean $\langle c z\rangle=11430 \mathrm{~km} \mathrm{~s}^{-1}$ and $\sigma_{v} \simeq 250 \mathrm{~km} \mathrm{~s}^{-1}$, are background galaxies either completely unrelated to the group, or they are infalling to the group. The radial-velocity difference between the group and the possible background double is again $\sim 900 \mathrm{~km} \mathrm{~s}^{-1}$, with a corresponding possible radial separation of $13 \mathrm{Mpc}^{2}$.

Our final sample consists of 23 groups (since HCG 50 is split into 2 groups) which almost all have in their environment accordant redshift galaxies. The groups HCG 35, HCG 58, HCG 60, and HCG 68 seem to be condensations within relatively rich groups consisting of $21,15,16$, and 15 members, respectively. Accordant redshift galaxies were not found in the environment of only one group, HCG 43. Note also that accordant redshift galaxies were found by de Carvalho et al. (1997) and Zabludoff \& Mulchaey (1998) in the nearest environment of 15 more HCGs. Thus, most of HGGs, together with the accordant redshift galaxies in their near environment, contain between 4 and $\sim 20$ galaxies, while their projected length reaches 1-2 Mpc.

\section{HCG and loose group association}

\subsection{Alignment of the HCG with its environment}

We find that the projected distribution of the accordant redshift galaxies in most cases lie along the position angle defined by the original HCG galaxies. In Fig. 2 we present the distribution of relative position angles, $\delta \phi$, between the original HCG position angle and that of the environment galaxies (in which case the original HCG is weighted only by its centre of mass). Similar alignment effects have been found between neighbouring clusters (e.g. Binggeli 1982; Plionis 1994), as well as between the major axis of the brightest cluster galaxies and that of the parent cluster itself (e.g. Plionis et al. 2003 and references therein). It is evident that accordant redshift galaxies are distributed preferentially along the elongation of the HCG (see also Tovmassian \& Tiersch 2001; Tovmassian 2001), implying group formation by infall and accretion along large-scale filaments.

The significance of the observed alignment signal is estimated by applying a Monte-Carlo procedure by which we ask

\footnotetext{
2 Including the original groups in our list only slightly changes the results of the forthcoming analysis (see Fig. 3).
} 


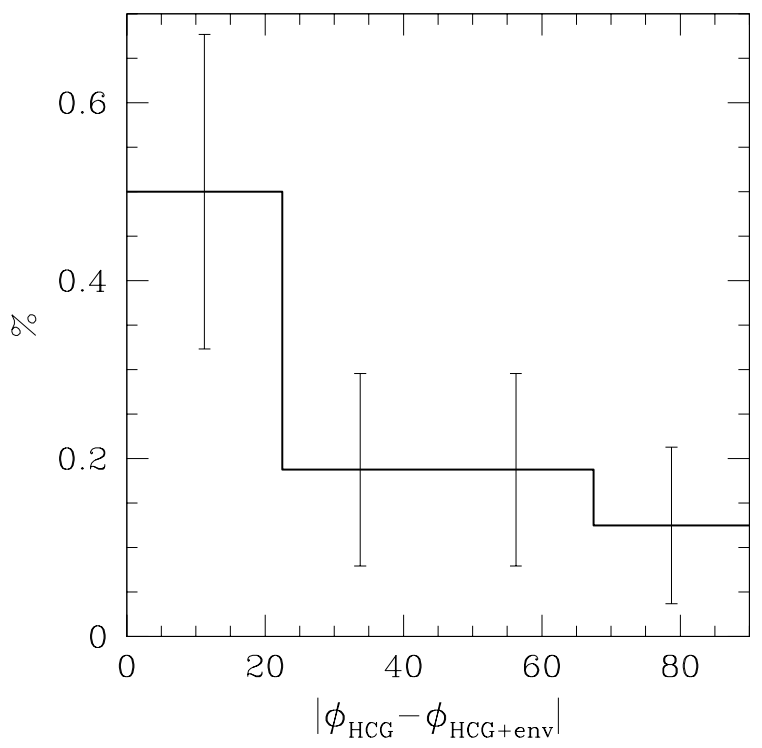

Fig. 2. The distribution of the misalignment angle between the HCG and its environment, where the latter includes the centroid of the HCG.

what probability there is that at least half of the $16 \delta \phi$ values, randomly selected between 0 and 90 degrees, will have $\delta \phi<22.5$, as observed in Fig. 2. We find that this probability is only 0.025 . This probability is also corroborated by estimating the first moment of the Fourier transfrom of the $\delta \phi$ distribution and comparing it with the expected random moment distribution. This approach gives a random probability of $\sim 0.029$, in rough agreement with our Monte-Carlo procedure.

\subsection{Velocity dispersion and the shape of HCGs and their environment}

In Table 1 we present the different parameters of the original HCGs and the corresponding values of the HCG + environment systems. The HCG designation is given in the 1 st column, the number of members and the velocity dispersion of the original HCG group are given in Cols. 2 and 3, respectively, while the corresponding values for the $\mathrm{HCG}+$ environment system are given in Cols. 4 and 5. Finally, the projected axial ratio of the latter systems is given in Col. 6.

We determined the axial ratio $q$ using the moment of inertia method (e.g. Carter \& Metcalfe 1980; Plionis et al. 2004). The HCG + environment systems with $N_{m} \leq 10$ have $\langle q\rangle \simeq$ $0.29 \pm 0.09$, similar to the mean elongation found by Plionis et al. (2004) for the corresponding UZC-SSRS2 group catalogue (USGC; Ramella et al. 2002), which is based on the Updated Zwicky Catalogue (UZC; Falco et al. 1999) and the Southern Sky Redshift Survey (SSRS2; da Costa et al. 1998). Hence, we may assume that they have a prolate-like shape similar to poor groups (see also Malykh \& Orlov 1986).

Inspecting Table 1 we see that the velocity dispersion of $\mathrm{HCG}+$ environment systems does not differ significantly from that of the original HCGs. In some HCG + environment systems, their $\sigma_{v}$ is even smaller than that of the original CGs, supporting the suggestion (Tovmassian \& Chavushyan 2000; Tovmassian 2002) that LG members are gravitationally bound to corresponding CGs. Thus, HCGs appear to be condensations in richer and gravitationally bound galaxy aggregates.

We also compare the mean axial ratio for all the $\mathrm{HCG}+$ environment systems (which is $\langle q\rangle=0.44_{-0.23}^{+0.10}$ ) with that
Table 1. Parameters of CGs and HCG + environment systems.

\begin{tabular}{|c|c|c|c|c|c|}
\hline HCG & $N_{m}$ & $\begin{array}{l}\sigma_{v} / \mathrm{km} \mathrm{s}^{-1} \\
\mathrm{HCG}\end{array}$ & & $\begin{array}{l}\sigma_{v} / \mathrm{km} \\
\mathrm{j}+\mathrm{env}\end{array}$ & ${ }^{q}{ }^{q}$ \\
\hline 7 & 4 & 89 & 7 & 89 & 0.22 \\
\hline 25 & 4 & 61 & 8 & 97 & 0.18 \\
\hline 35 & 6 & 350 & 21 & 306 & 0.63 \\
\hline 40 & 5 & 149 & 6 & 296 & 0.55 \\
\hline 43 & 5 & 222 & 5 & 222 & 0.61 \\
\hline 45 & 4 & 234 & 10 & 381 & 0.66 \\
\hline 49 & 4 & 34 & 9 & 60 & 0.19 \\
\hline 50 & 5 & 472 & 8 & 546 & - \\
\hline $50 \mathrm{a}$ & & - & 4 & 266 & 0.45 \\
\hline $50 \mathrm{~b}$ & & - & 4 & 96 & 0.16 \\
\hline 56 & 5 & 170 & 6 & 126 & 0.29 \\
\hline 58 & 5 & 162 & 15 & 140 & 0.46 \\
\hline 60 & 4 & 427 & 16 & 472 & 0.60 \\
\hline 63 & 3 & 132 & 7 & 188 & 0.21 \\
\hline 64 & 3 & 214 & 6 & 239 & 0.26 \\
\hline 66 & 4 & 306 & 9 & 414 & 0.78 \\
\hline 67 & 4 & 211 & 14 & 339 & 0.73 \\
\hline 68 & 5 & 172 & 15 & 155 & 0.65 \\
\hline 82 & 4 & 620 & 6 & 233 & 0.22 \\
\hline 87 & 3 & 121 & 6 & 215 & 0.19 \\
\hline 88 & 4 & 27 & 6 & 76 & 0.39 \\
\hline 92 & 4 & 390 & 10 & 337 & 0.53 \\
\hline 97 & 5 & 370 & 14 & 402 & 0.66 \\
\hline 98 & 3 & 120 & 8 & 238 & 0.30 \\
\hline
\end{tabular}

of the USGC groups. Due to the possibly intrinsic correlation of the group elongation with group membership, we weight the original USGC $q$ values such that they respect the membership distribution of the HCG + environment systems. Doing so, we find a USGC group axial ratio of $\langle q\rangle=0.436 \pm 0.09$, which completely agrees with that of the $\mathrm{HCG}+$ environment systems.

\subsection{Correlation of velocity dispersion, number of group members, and shape}

Compact groups, as well as poor groups of galaxies, have been shown to have prolate-like shapes (Malykh \& Orlov 1986; Oleak et al. 1995; Plionis et al. 2004), while their observed $\sigma_{v}$ appears to depend on the orientation of the group with respect to the observer (e.g. Tovmassian et al. 1999). The similarity of the axial ratio distribution and its mean value between the original HCGs and the sample of HCG + environment systems studied here and in the work of Tovmassian \& Chavushyan (2000) suggests that the HCG + environment systems also have a prolate-like shape.

Therefore, if galaxies move in radial orbits along the major axis of these highly elongated groups, one should on the basis of projection effects expect a correlation between the velocity dispersion of the groups and their axial ratio, by which groups oriented close to the orthogonal to the line of sight will have relatively small projected $q$ and small $\sigma_{v}$ while groups oriented close to the line of sight will have larger $q$ 's and larger $\sigma_{v}$ 's.

Indeed, for the considered HCG + environment systems, we find a strong $(R=0.72)$ and significant $\left(P_{\text {rand }} \simeq 10^{-4}\right)$ correlation between $\sigma_{v}$ and $q$ (upper panel of Fig. 3) with:

$\sigma_{v} / \mathrm{km} \mathrm{s}^{-1}=403( \pm 91) q+61( \pm 44)$.

This effect can also be seen if we divide the groups into those with $\sigma_{v} \geq 220 \mathrm{~km} \mathrm{~s}^{-1}$ (13 groups) and those with $\sigma_{v}<$ $220 \mathrm{~km} \mathrm{~s}^{-1}$ (10 groups), which have a median $q$-value and $67 \%$ and $33 \%$ quantile limits of $0.6_{-0.15}^{+0.01}$ and $0.21_{-0.02}^{+0.01}$, respectively. 

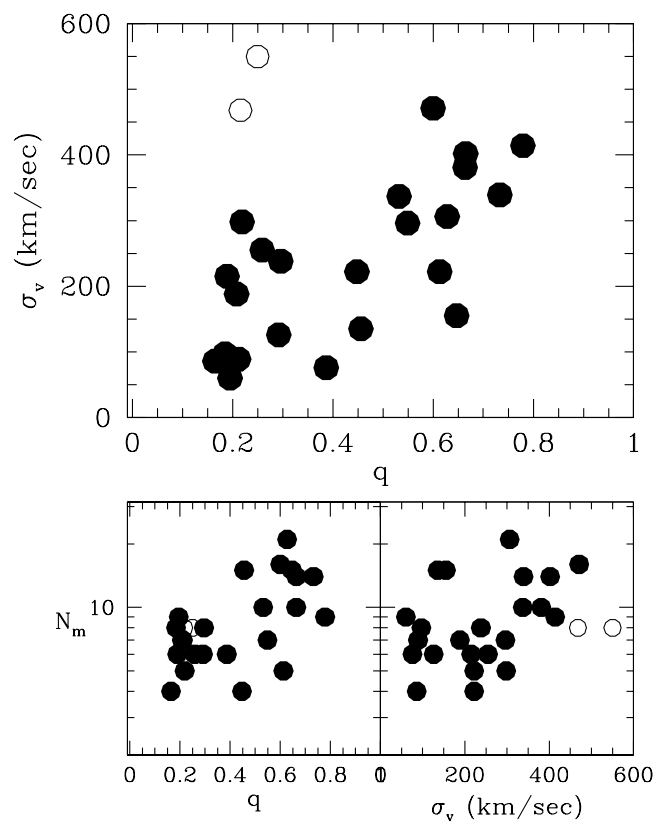

Fig. 3. Correlation between $\sigma_{v}$ and $q$ (upper panel), $q$ and $N_{m}$ (lower left panel), and $\sigma_{v}$ and $N_{m}$ (lower right panel). The open symbols represent the HCG + environment groups containing the original HCG50 and 82, assumed to be the result of projection effects (see Sect. 3.1). However, the $q-\sigma_{v}$ correlation persists even if we include these two groups in their original form, but it is weaker $\left(R=0.44\right.$ and $\left.P_{\text {rand }}=0.03\right)$.

However, beyond the orientation effect discussed, another effect could contribute or even dominate the observed correlation. This is related to the possibly different evolutionary stage of groups with different values of $q$. Richer systems (with larger $N_{m}$ ) will be relatively more virialized, with a higher value of $\sigma_{v}$, and will therefore have a higher value of $q$, since virialization tends to sphericalize dynamical systems. The lower left panel of Fig. 3 shows that groups with larger $N_{m}$ indeed have a higher axial ratio $q$, with quite a strong correlation $\left(R=0.57, P_{\text {rand }}=\right.$ $0.005)$. Since $\sigma_{v}$ is also only weakly $\left(R=0.43, P_{\text {rand }}=0.05\right)$ correlated with $N_{m}$ (lower right panel of Fig. 3), different stages of virilization cannot be the sole reason for the $\sigma_{v}-q$ correlation, so the orientation effect should be a major contributer.

As a further test of the relative strength of the two effects (orientation and virialization) we study the corresponding correlation of the 17 groups with $N_{m} \leq 10$, which are relatively poor and thus are not expected to be at a very different dynamical stage. Indeed, as can be seen in Fig. 4 (lower left and right panels) for these groups, we find no $q-N_{m}$ or $\sigma_{v}-N_{m}$ correlations $\left(R \simeq 0.25\right.$ and $P_{\text {rand }} \simeq 0.3$ for both), while we do find a strong $q-\sigma_{v}$ correlation (upper panel of Fig. 4) with $R=0.69$ and $P_{\text {rand }}=0.002$.

However, we would like to caution the reader that it is also possible that groups of even equal mass could be at different dynamical stages, depending on the environment in which they are located. Indeed Ragone \& Plionis (2006, in preparation), used large cosmological $N$-body simulations to find that there is a significant correlation between the velocity dispersion and the group elongation (in 3D space but also surviving in projection) for halos of equal mass.

Finally, we investigate whether discreteness effects can introduce a bias in the correlations discussed so far. Tracing ellipsoids with a discrete distribution of particles can bias the intrinsic shape of the parent structure (e.g. Paz et al. 2006). Projection from $3 \mathrm{D}$ to $2 \mathrm{D}$ also alters the intrinsic axial ratio distribution
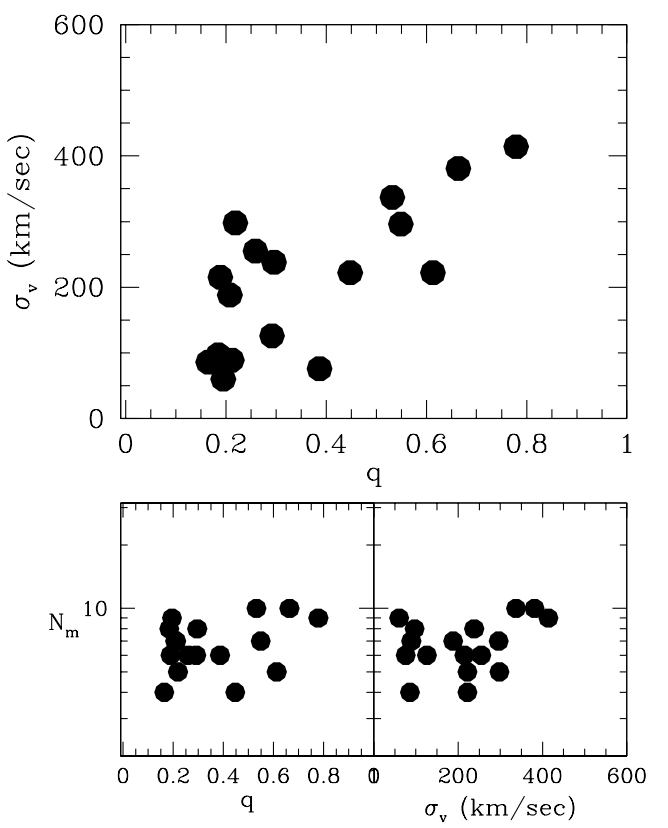

Fig. 4. As in Fig. 3 but for the poorest of the HCG + environment groups $\left(4 \leq N_{m} \leq 10\right)$.
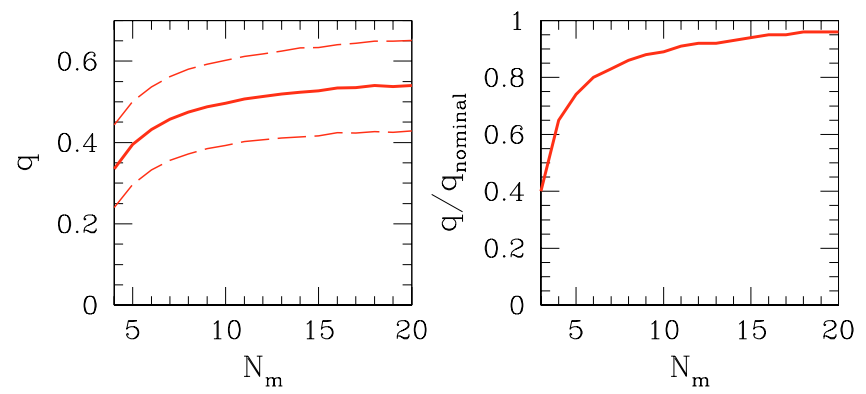

Fig. 5. Left panel: the projected mean axial ratio of Monte-Carlo prolate groups with an intrinsic mean axial ratio of 0.43 as a function of points used to sample the group. Right panel: the corresponding fractional decrease of the projected axial ratio as a function of the number of points used to sample the group.

(cf. Hubble 1926; Carter \& Metcalfe 1980) with the projected structures appearing more spherical and typically smaller than the 3D ones. We investigated the coupling of the two effects by Monte-Carlo simulations. We traced a 3D prolate spheroid of predetermined axial ratio and size with a variable number of particles. We then projected the distribution of particles in the three Cartesian planes after randomly orienting the spheroids with respect to a predetermined line of sight.

In the left panel of Fig. 5 we present the median projected $q$ values together with the $67 \%$ and $33 \%$ quantile limits, derived from our Monte-Carlo analysis, as a function of the number of points used to sample the 3D prolate spheroid. We have used an intrinsic axial ratio for the $3 \mathrm{D}$ prolate spheroid of $\beta=0.43$, which provides a projected axial ratio of $\sim 0.5$, near the value of our best-sampled (richer) $\mathrm{HCG}+$ environment groups.

It is evident that there is a trend toward decreasing sphericity with decreasing group galaxy membership as in the observed group case. In the right panel of Fig. 5 we show the fractional decrease in the nominal projected group axial ratio, which in turn provides us with a multiplicative correction factor by which we can attempt to crudely correct the observed $q$ group values for the effect of discreteness. Small variations in the input 
nominal group $q$-value changes the fractional correction factor insignificantly.

We again quantify the previous correlations but now using the corrected group $q$ values, and we find that the $q-\sigma_{v}$ correlation remains mostly unaltered and significant $(R=0.63$ and $\left.P_{\text {rand }}=0.001\right)$, while the $q-N_{m}$ correlation, which could be viewed as that resulting from the different virialization stages of groups of different richness, becomes weak and completely insignificant $\left(R=0.25\right.$ and $\left.P_{\text {rand }}=0.26\right)$.

These results indicate that the orientation effect could be the dominant one in shaping the $q-\sigma_{v}$ correlation. As a final point, the dynamical properties and correlations of HCG + environment systems do not differ from those of poor groups.

\subsection{The $M_{K}$ magnitudes of E,SO galaxies in HCGs}

Tovmassian et al. (2005, hereafter TPA) found that the mean $K$-band absolute magnitude $\left\langle M_{K}\right\rangle$ of E/S0 galaxies in USGC poor groups, located at distances $1000<c z<5500 \mathrm{~km} \mathrm{~s}^{-1}$, is equal to -23 . 42 . This is brighter by 0.75 on average than that of isolated E/S0 galaxies and of spiral galaxies (Sa and later) either in groups or in the field (in the same redshift range and within the same - within 0.2 mags - apparent magnitude limit). Furthermore, the $\left\langle M_{B}\right\rangle$ values of isolated $\mathrm{E} / \mathrm{S} 0$ and spiral galaxies practically do not differ from each other. This fact allowed TPA to conclude that E/S0's in groups are formed as the result of the merging of two galaxies of comparable luminosity and thus mass (if we assume the same $M / L$ ).

In order to perform a similar study using CGs, we selected all HCGs that lie in the previously mentioned redshift range and determined absolute $K$-band magnitudes, as in TPA, by using the $K_{\mathrm{s} \text {-total }}$ magnitudes from $2 M A S S$ (see Jarrett et al. 2000; http://www.ipac.caltech.edu/2mass), corrected for Galactic extinction according to Schlegel et al. (1998). Furthermore, the group radial velocities were corrected for the peculiar velocity of the Local Group and a local velocity field that contains a Virgo-centric infall component and a bulk flow given by the expectations of linear theory (see Branchini et al. 1996).

We do not restrict our HCGs sample to that of Table 1 because most of these HCGs have redshifts outside the range of interest. We extend our sample to include all HCGs that fall within this range (in total 20 groups) and therefore in the estimation of the mean absolute galaxy luminosities we use only HCG galaxies and not accordant redshift galaxies of the environment (which we have identified only for the HCGs which fall within the SDSS survey limits).

It is important to note that the magnitude limits of the galaxy samples from which the USGC and HCG groups have been selected, are different ( $m_{B} \sim 15.5$ and $\sim 17$, respectively). This implies that the two galaxy samples will trace a different fraction of intrinsically faint and bright galaxies. The HCG galaxies being drawn from a fainter sample than the USGC sample will contain a higher fraction of nearby faint galaxies. However, we can overcome the bias introduced by (a) excluding from our comparison the HCG galaxies with apparent magnitudes that are larger than the USGC limit $\left(m_{B} \sim 15.5\right)$ and (b) limiting our analysis to the same redshift range and requiring that the two samples have statistically compatible redshift distributions. In Fig. 6 we present the redshift distribution of the two group samples and indeed, we verify that this is the case since the KS two-sampled test shows that the probability of the two redshift distributions have been drawn from the same parent distribution is 0.15 .

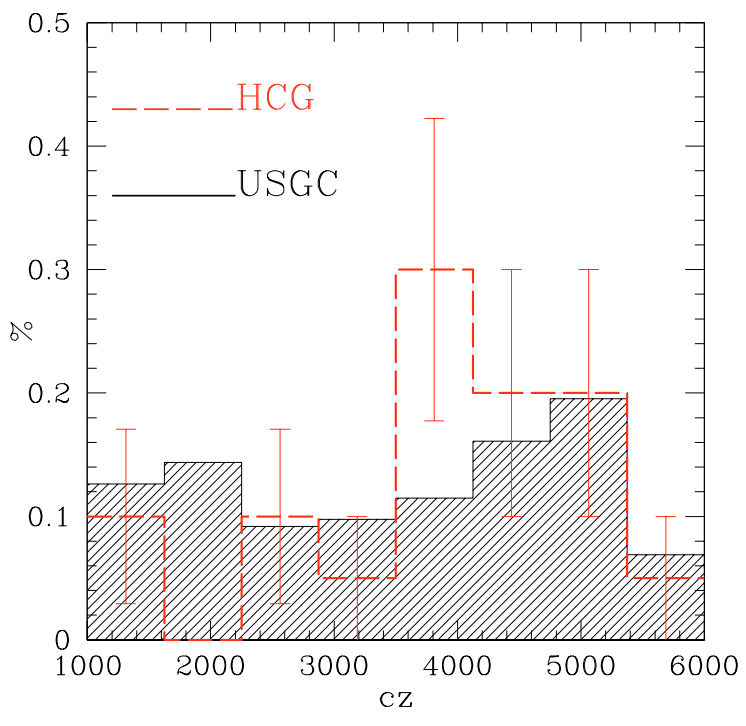

Fig. 6. The redshift distribution of the USGC and HCG groups in the range $1000<c z<5500 \mathrm{~km} \mathrm{~s}^{-1}$.

Table 2. The mean absolute magnitudes $\left\langle M_{K}\right\rangle$ of E/S0 and spiral galaxies in HCG and poor USGC groups. The quoted uncertainty is the distribution's standard deviation. The galaxy numbers are shown in parenthesis.

\begin{tabular}{lcc}
\hline \hline & E/S0 & Spirals \\
\hline USGC & $-23.83 \pm 1.18(156)$ & $-22.96 \pm 1.25(310)$ \\
HCG & $-23.79 \pm 1.12(24)$ & $-22.72 \pm 1.39(24)$ \\
\hline
\end{tabular}

In Table 2 we compare the $\left\langle M_{K}\right\rangle$ values for the USGC and HCG groups within the same radial-velocity limits and with $m_{B} \lesssim 15.5$. It is evident that the corresponding average absolute magnitudes of early type galaxies between the USGC and HCG groups are almost identical $\left(\delta M_{K} \sim 0.04\right)$, from which we infer that in both HCG and USGC groups, the E/S0 galaxies are formed similarly by the merging of two spiral galaxies of roughly the same luminosity (see TPA).

A more complete study of the possible luminosity differences of the two group samples would entail deriving their respective luminosity functions (e.g., Mendes de Oliveira \& Hickson 1991; Kelm \& Focardi 2004), which is however beyond the scope of the present study.

\subsection{The fraction of E/SO galaxies}

TPA also found that the mean fraction of E/S0 galaxies of poor USGC groups with $4 \leq N_{m} \leq 10$ is $\left\langle f_{\mathrm{E} / \mathrm{S} 0}\right\rangle \sim 0.23$, significantly higher than that of the Karachentseva et al. (1986) sample of isolated galaxies, which is equal to $\sim 0.15$. They also showed that $f_{\mathrm{E} / \mathrm{S} 0}$ depends very weakly on the group's richness, while a significant, although weak, correlation exists between the group $f_{\mathrm{E} / \mathrm{S} 0}$ and velocity dispersion.

In this work we use the morphological classification of HCG member galaxies from Hickson (1994). Note that we have no such classification for the accordant redshift galaxies, so we only consider the HCG galaxies in this section. We caution the reader that this may not properly represent the morphological content of the whole $\mathrm{HCG}+$ environment system.

The mean fractions of E/S0 galaxies in the HCG groups is $\left\langle f_{\mathrm{E} / \mathrm{S} 0}\right\rangle \sim 0.43$ (with the corresponding median of 0.4 ), which is about twice the value of the USGC poor groups, which in turn 


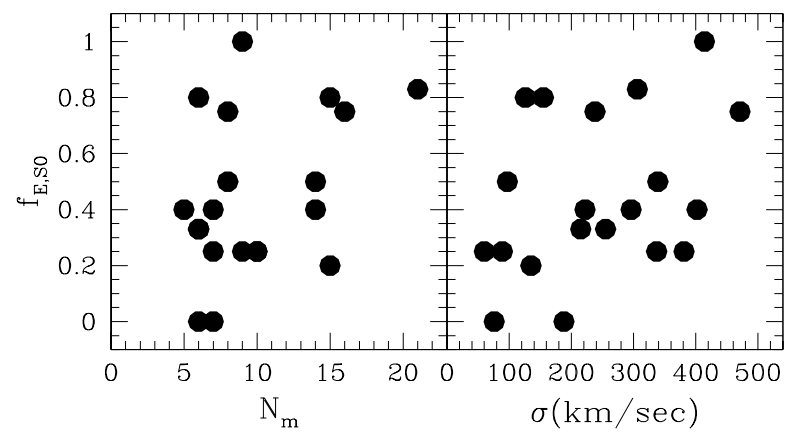

Fig. 7. Correlation of the fraction $f_{\mathrm{E} / \mathrm{S} 0}$ with $N_{m}$ (left panel) and with $\sigma_{v}$ (right panel) for the $\mathrm{HCG}+$ environment groups.

is $\sim 65 \%$ higher than that of isolated galaxies (Tovmassian et al. 2004). Kelm \& Focardi (2004) have also found that the UZC CGs have a higher content of E/S0's than found among isolated galaxies. They also showed that the fraction of E/SO's among close neighbours of CGs is intermediate between that of CGs and isolated galaxies.

Furthermore, we find a weak correlation between $\mathrm{HCG}+$ environment richness $\left(N_{m}\right)$ and $f_{\mathrm{E} / \mathrm{s} 0}$ (with $R=0.36$ and random probability $P=0.11$; see left panel of Fig. 7). For example, the median value $f_{\mathrm{E} / \mathrm{S} 0}$ of groups with $N_{m} \leq 8$ is equal to $0.33_{-0.03}^{+0.07}$, while the corresponding value of the groups with $N_{m} \geq 9$ is $0.40_{-0.15}^{+0.10}$. This correlation, albeit weak, seems to agree with the well-established morphology-density relation (e.g. Dressler 1980; Goto et al. 2003; Helsdon \& Ponman 2003).

We also find a weak correlation between $\sigma_{v}$ and $f_{\mathrm{E} / \mathrm{S} 0}$ with $R=0.37$ and $P_{\text {rand }}=0.1$ (see right panel of Fig. 7). For example, dividing the HCG + environment groups in two subsamples based on their velocity dispersion, we find $\left\langle f_{\mathrm{E} / \mathrm{S} 0}\right\rangle=0.40_{-0.15}^{+0.10}$ for those with $\sigma_{v} \geq 220 \mathrm{~km} \mathrm{~s}^{-1}$ and $\left\langle f_{\mathrm{E} / \mathrm{S} 0}\right\rangle=0.25_{-0.15}^{+0.08}$ for those with $\sigma_{v} \leq 220 \mathrm{~km} \mathrm{~s}^{-1}$.

Using the sample of richer LGs in the environment of some poor groups and HCGs, Zabludoff \& Mulchaey (1998) also find that $f_{\mathrm{E} / \mathrm{S} 0}$ is definitely correlated with $\sigma_{v}$. In order to explain the $f_{\mathrm{E} / \mathrm{S} 0}-\sigma_{v}$ correlation, they suggest that either galaxy morphology is set by the size of the local potential at the time of galaxy formation (Hickson et al. 1988) and/or that $\sigma_{v}$ and $f_{\mathrm{E} / \mathrm{s} 0}$ increase as a group evolves (Diaferio et al. 1995). The correlation, albeit weak, between $f_{\mathrm{E} / \mathrm{S} 0}$ and the HCG X-ray luminosity (Ponman et al. 1996) also suggests that groups with high values of $f_{\mathrm{E} / \mathrm{S} 0}$ are dynamically more evolved, possibly virialized systems.

In line with this model, Tovmassian et al. (2004) suggested that in elongated, prolate-like groups in which members move predominantly along the group elongation, the probability of encounter and merging, and thus formation of E/S0 galaxies, is higher than in a spherical overdensity of the same major axis size in which galaxy orbits are "chaotic". In effect what we suggest is that in such highly elongated groups and prior to virialization, galaxies move along the group elongation in semi-regular orbits, as expected during the anisotropic infall of galaxies in groups/clusters along filaments. During this phase and due to the initially lower velocity dispersion of these unrelaxed systems, many interactions and merging take place (from which the higher fraction of E/S0 emerges). Subsequently, the velocity dispersion of these systems increases due to virialization processes, which also results in heating the intergalactic gas, which then emits in X-rays.

\subsection{The fraction of active galaxies}

The high-density environment of the HCGs, in combination with their relative low-velocity dispersion suggests that these environments are probable sites of strong galaxy interactions, also suggested by their higher fraction of E/S0 galaxies. If the AGN fueling mechanism is triggered by such interactions, one would expect to see a different fraction of AGNs with respect to the field. Kelm et al. (1998) find that $3 \%$ of HCG members are Seyferts, while if one includes LINERs this fraction increases to $\sim 18 \%$ (Coziol et al. 2000). Furthermore, the latter authors show that when including low-luminosity systems, this fraction increases even further to $\sim 40 \%$, in agreement with Ho et al. (1997).

For our sample of 14 HCGs, we have spectral classification for $32 \mathrm{HCG}$ galaxies and 87 close environment galaxies. Since we do not have the spectral classification of all the galaxies in the HCGs and their environment, we stack all the available data together in two samples (HCG and close environment galaxies). Furthermore, the different distances of the HCGs analysed in this work produce a variable sampling of the individual luminosity function of the different HCGs and their environment. Therefore, the following analysis is mostly valid as a comparison between the activity in HCGs and their close environment.

It is interesting that no Seyfert 1 spectra were identified, in agreement with the analysis of Coziol et al. (2000) of the Southern CG sample (Iovino 2002). However, in our case, we cannot exclude that small number statistics could be the cause of such a paucity. Most importantly, we find $31 \%( \pm 10 \%)$ and $13 \%( \pm 6 \%)$ of AGNs and starburst galaxies, respectively, in the HCGs, while these fractions are $29 \%( \pm 6 \%)$ and $4.6 \%( \pm 2.3 \%)$ for the close environment galaxies. If we restrict our analysis to Seyfert-type AGNs (excluding LINERs), we find that their fractions are $6.5 \%$ and $8 \%$ respectively between the HCGs and their close environment. These fractions generally agree with Coziol et al. (2000), but the Seyfert fraction appears to be higher than that of Kelm et al. (2004).

The most important results of this analysis are that

- the fraction of AGNs (being Seyferts or LINERs) does not vary significantly between the HCGs and the looser groups within which they are embedded (see also Shimada et al. 2000); and

- what does vary at a significant level appears to be the fraction of starbursting galaxies, which is higher in the dense environment of the HCGs.

The former could be the result of perturbations caused by the HCG tidal field on disk galaxies, even before close galaxy encounters and interactions take place in the HCG centres. Such perturbations can cause an inflow of gas towards the disk centre, triggering bursts of star formation and possibly feeding the AGN (e.g. Byrd \& Valtonen 1990; Fujita 1998). This could explain why the fraction of AGNs is the same in the HCGs and in their close environment, implying that the AGNs are already well in place in the looser groups. The higher fraction of starbursting galaxies in the HCGs with respect to their close environment then implies that only the starburst activity is enhanced by the close encounters and interactions that take place in the dense HCGs.

Furthermore, Focardi \& Kelm (2002) and Coziol et al. (2004) have found a correlation between the dynamics of the CG and the galaxy member activity, with galaxies being more active and dominated by late-type morphologies in lowervelocity dispersion systems. We also verify their result in our 


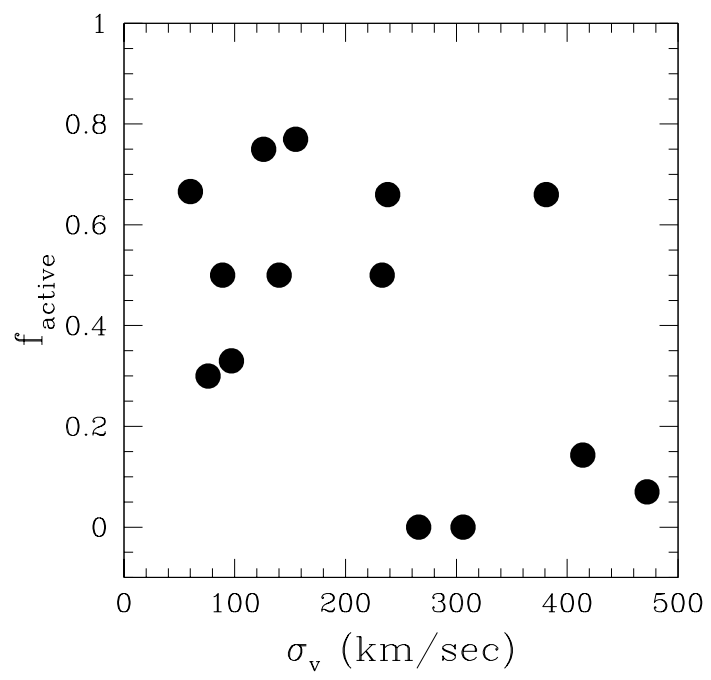

Fig. 8. Correlation of the fraction of active $\mathrm{HCG}+$ environment galaxies, $f_{\text {active }}$, with the HCG + environment velocity dispersion.

the $\mathrm{HCG}+$ environment systems, as can be seen in Fig. 8, where we correlate the fraction of active galaxies (AGNs and starbursts) with the $\mathrm{HCG}+$ environment velocity dispersion. An anti-correlation is present with Spearman correlation coefficient of $R=-0.45$ and the probability of random correlation being $P=0.12$. This anti-correlation is robust to the exclusion of individual groups.

A possible overall picture thus emerges in which highvelocity dispersion CGs, which are also X-ray luminous (e.g. Ponman et al. 1996), are dynamically more evolved and their late-type galaxy members had enough time to interact, merge, and evolve into earlier types. The low-velocity dispersion CGs are dynamically younger, richer in late-type and in active galaxies and yet unevolved.

\section{Conclusions}

Using SDSS spectroscopic data, we searched the close environment of 22 HCGs and found that the large majority is embedded in galaxy overdensities that extend up to $\sim 1 \mathrm{Mpc}$ around the HCG centre. A wealth of indications point in the direction that HCGs are condensations within looser prolate-like groups of galaxies with dynamical and morphological characteristics similar to those of poor groups (like the USGC groups studied by Plionis et al. 2004).

These include: (a) the HCG + environment elongation is similar to that of the USGC groups; (b) the HCG + environment groups show the same axial ratio - velocity dispersion correlation as the USGC groups, a correlation which could be due to an orientation effect of intrinsically prolate-like groups; (c) the mean $K$-band absolute magnitude $\left\langle M_{K}\right\rangle$ of E/S0 galaxies in HCGs is about the same as that in poor USGC groups, which is brighter by $\sim 0$. 75 than that of isolated E/S0's and group or isolated spiral galaxies, showing that E/S0's in CGs, as in USGC groups, could be formed as the result of the merging of two galaxies of similar luminosity; (d) the mean fraction of early type galaxies in HCGs is higher than in the field but also twice as high as in poor USGC groups, indicating enhanced merging in the central compact condensations of poor groups; (e) the fraction $f_{\mathrm{E} / \mathrm{S} 0}$ depends weakly on the richness and the velocity dispersion of the HCG + environment groups; (f) the fraction of AGNs is similar between members of HCGs and their close environment galaxies, while the fraction of starburst galaxies is significantly higher in the HCGs; (g) the fraction of active galaxies (AGNs and starbursts) is anti-correlated with the velocity dispersion of the HCG + environment systems.

These results could be explained by noting that groups are elongated prolate-like structures (e.g. Plionis et al. 2004), in which galaxies should move in radial orbits around the group gravitational centre. Thus we expect that what appears on many occasions to be a CG is just an ordinary group, or part of it, observed either when some of its member galaxies happen to be close to one another, during their radial orbits along the group elongation, or if the group is oriented close to the line of sight and all its members are projected over a small solid angle. Of course in the former case, gravity will play its role in inducing interactions, the results of which will depend on a multitude of initial conditions (relative galaxy velocities, orbit trajectories, local background density, galaxy morphology, gas content, etc.). Our results indicate that the main outcome of such interactions in the CG centre is an enhanced starburst activity but not necessarily the triggering of an AGN.

Furthermore, our results show that the members of groups with high-velocity dispersion are predominantly early-type nonactive galaxies, while members of presently low-velocity groups (probably indicating their earlier evolutionary stage) are predominantly late-type active galaxies. These findings are in good agreement with Coziol et al. (2004) and suggest an evolutionary sequence by which initially late-type galaxies, in an initially low-velocity dispersion group (indicating either a low-mass system or a dynamically unevolved, more massive system), interact with the group tidal field - by which both the AGN and starburst activity is triggered - then galaxy merging and virialization processes, which increase the group velocity dispersion and X-ray emission, will lead to a high-velocity dispersion, $\mathrm{X}$-ray luminous group with a higher fraction of early-type galaxies. Note also that numerical simulations (Gómez-Flechoso \& Dominguez-Tenreiro 2001) have shown that there is a lack of enhanced merger activity in the quiescent virialized CG halos, suggesting that early-type galaxies form in the initial group formation stage, when the CG velocity dispersion is still low.

Acknowledgements. H.M.T. and M.P. acknowledge funding by the Mexican Government grants SEP-2003-C02-44376/A-1 and CONACyT-2002-C0139679 , respectively. We thank the referee, Gary Mamon, for many useful comments and suggestions.

\section{References}

Abazajian, K., Adelman-McCarthy, J. K., Agüeros, M. A., et al. 2005, AJ, 129, 1755

Baier, F. W., \& Tiersch, H. 1979, Astrofizika, 15, 33

Barnes, J. 1985, MNRAS, 215, 517

Barnes, J. 1989, Nature, 338, 123

Barnes, J. 1990, in Dynamics and Interaction of Galaxies, ed. Weilen (SpringerVerlag, Heidelberg), 186

Binggeli, B. 1982, A\&A, 107, 338

Bode, P. W., Cohn, H. N., \& Lugger, P. M. 1993, ApJ, 416, 17

Branchini, E., Plionis, M., \& Sciama, D. W. 1993, ApJ, 461, L17

Byrd, G., \& Valtonen, M. 1990, ApJ, 350, 89

Carter, D., \& Metcalfe, N. 1980, MNRAS, 191, 325

Coziol, R., Iovino, A., \& de Carvalho, R. R. 2000, AJ, 120, 47

Coziol, R., Brinks, E., \& Bravo-Alfaro, H. 2004, AJ, 128, 68 [CBBA]

da Costa, L. N., Willmer, C. N. A., Pellegrini, P. S., et al. 1998, AJ, 116, 1

de Carvalho, R. R., Ribeiro, A. L. B., \& Zepf, S. E. 1994, ApJS, 93, 47

de Carvalho, R. R., Ribeiro, A. L. B., Capelato, H. V., \& Zepf, S. E. 1997, ApJS, 110,1

de Carvalho, R. R., Gonçalves, T. S., Iovino, A., et al. 2005, AJ, 130, 425

Diafero, A., Geller, M. J., \& Ramella, M. 1994, AJ, 107, 868

Diafero, A., Geller M. J., \& Ramella, M. 1995, AJ, 109, 2293

Dressler, A. 1980, ApJ, 236, 351 
Falco, E. E., Kurtz, M. J., Geller, M. J., et al. 1999, PASP, 111, 438

Focardi, B., \& Kelm, B. 2002, A\&A, 391, 35

Fujita, Y. 1998, ApJ, 509, 587

Gómez-Flechoso, M. A., \& Dominguez-Tenreiro, R. 2001, ApJ, 549, L187

Goto, T., Yamauchi, C., Fujita, Y., et al. 2003, MNRAS, 346, 601

Governato, F., Tozzi, P., \& Cavaliere, A. 1996, ApJ, 458, 18

Hubble, E. P. 1926, ApJ, 64, 321

Jarrett, T. H., Chester, T., Cutri, R, et al. 2000, AJ, 119, 2498

Hickson, P. 1982, ApJ, 255, 382

Hickson, P. 1994, Atlas of Compact Groups of Galaxies (New York: Gordon and Breach)

Hickson, P. 1997, ARA\&A, 35, 360

Hickson, P., Huchra, L., \& Kindl, E. 1988, ApJ, 331, 64

Hickson, P., Ninkov, Z., Huchra, P., \& Mamon, G. A. 1984, in Clusters and Groups of Galaxies, ed. F. Mardirossian, G. Guiricin, \& Mezzetti, 367

Hickson, P., Mendes de Oliveira, C., Huchra, J. P., \& Palumbo, G. G. C. 1992, ApJ, 399, 353

Ho, L. C., Filippenko, A. V., \& Sargent, W. L. W. 1997, ApJ, 487, 568

Iovino, A. 2002, AJ, 124, 2471

Iovino, A., de Carvalho, R. R., Gal, R. R., et al. 2003, AJ, 125, 1660

Karachentseva, V. E. 1973, Comm. Spec. Ap. Obs. USSR, 8, 1

Kelm, B., \& Focardi, P. 2004, A\&A, 418, 937

Kelm, B., Focardi, P., \& Palumbo, G. G. C. 1998, A\&A, 335, 912

Kelm, B., Focardi, P., \& Zitelli, V. 2004, A\&A, 418, 25

Kuntschner, H., Smith, R. J., Colles, M., et al. 2002, MNRAS, 337, 172

Lee, B. C., Allam, S. S., Tucker, D. L., et al. 2004, AJ, 127, 1811

Malykh, S. A., \& Orlov, V. V. 1986, Astofizika, 24, 445

Mamon, G. A. 1987, ApJ, 321, 622

Mamon, G. A. 1990, IAU Coll., 124, 609

Mamon, G. A. 1992, ApJ, 401, L3

Mamon, G. A. 2000, IAU 174 on Small Galaxy Groups, ed. M. Valtonen, \& C. Flynn, ASP Ser., 209, 217

Marzke, R. O., Geller, M. J., Huchra, J. P., \& Corwin, H. G. Jr. 1994, AJ, 108, 437

Mendes de Oliveira, C., \& Hickson, P. 1991, ApJ, 380, 30

Mendes de Oliveira, C., \& Hickson, P. 1994, ApJ, 427, 684
Moles, M., del Olmo, A., Perea, Masegosa, J., Marquez, I, \& Costa, V. 1994, A\&A, 285, 404

Oleak, H., Stoll, D., Tiersch, H., \& MacGillivray, H. T. 1995, AJ, 109, 1485

Paz, D. J., Lambas, D. G., Padilla, N., \& Merchán, M. 2006, MNRAS, 366, 1503 Plionis, M. 1994, ApJS, 95, 401

Plionis, M., Benoist, C., Maurogordato, S., Ferrari, C., \& Basilakos, S. 2003, ApJ, 594, 144

Plionis, M., Basilakos, S., \& Tovmassian, H. M. 2004, MNRAS, 352, 1323

Ponman, T. J., Bourner, P. D. J., Ebeling, H., \& Bohringer, H. 1996, MNRAS, 283,690

Ramella, M., Diaferio, A., Geller, M. J., \& Huchra, J. P. 1994, AJ, 107, 1623

Ramella, M., Geller, M. J., Pisani, A., \& da Costa, L. N. 2002, AJ, 2976, 123

Ribeiro, A. L. B., de Carvalho, R. R., Capelato, H. V., \& Zepf, S. E. 1998, ApJ, 497,72

Rose, J. A. 1977, ApJ, 211, 1927

Rood, H. J. 1979, ApJ 233, 21

Rood, H. J., \& Struble, M. F. 1994, PASP, 106, 416

Rood, H. J., \& Williams, B. A. 1989, ApJ, 339, 772

Schlegel, D. J., Finkbeiner, D. P., \& Davis, M. 1998, ApJ, 500, 525

Shakhbazian, R. K. 1973, Astrofizika, 9, 495

Shimada, M., Ohyama, Y., Nishiura, S., et al. 2000, AJ, 119, 2664

Shostak, G. S., Sullivan III, W. T., \& Allen, R. J. 1984, A\&A, 139, 15

Sulentic, J. W. 1987, ApJ, 322, 605

Tovmassian, H. M. 2001, PASP, 113, 543

Tovmassian, H. M. 2002, AN, 323, 488

Tovmassian, H. M., \& Chavushyan, V. H. 2000, AJ, 119, 1687

Tovmassian, H. M., \& Tiersch, H. 2001, A\&A, 378, 740

Tovmassian, H. M., Martinez, O., \& Tiersch, H. 1999, A\&A, 348, 693

Tovmassian, H. M., Yam, O., \& Tiersch, H. 2001, Rev. Mex. A\&A, 37, 173

Tovmassian, H. M., Plionis, M., \& Andernach, H. 2004, ApJ, 617, L111 [TPA]

West, M. J. 1989, ApJ, 344, 535

Zabludoff, A. I., \& Mulchaey, J. S. 1998, ApJ, 496, 39

Zepf, S. E. 1993, ApJ, 418, 72

Zepf, S. E., Whitmore, B. C., \& Levison, H. F. 1991, ApJ, 383, 524

Vennik, J., Richter, G. M., \& Longo, G. 1993, AN, 314, 393

Williams, B. A., Yun, M. S., \& Verdes-Montenegro, L. 2002, AJ, 123, 2417 
H. Tovmassian et al.: Hickson compact groups and their environment, Online Material $p 1$

\section{Online Material}


H. Tovmassian et al.: Hickson compact groups and their environment, Online Material p 2
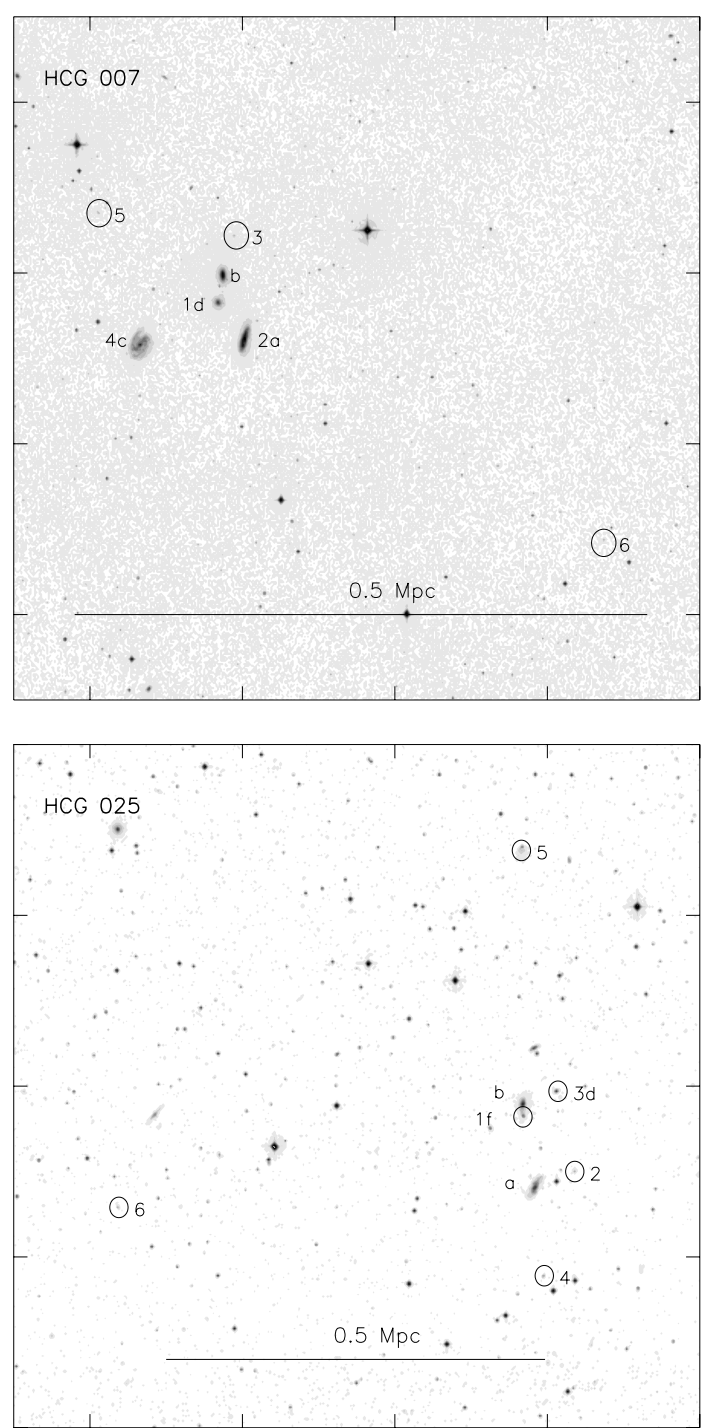

Fig. 1. Digital Sky Survey images of the the environment of HCGs Original HCG members are marked by letters $(a, b$, etc.), as in Hickson (1994), while the SDSS galaxies are marked by circles and they are numbered $(1,2,3$, etc.). Accordant redshift galaxies are those marked only by a number.
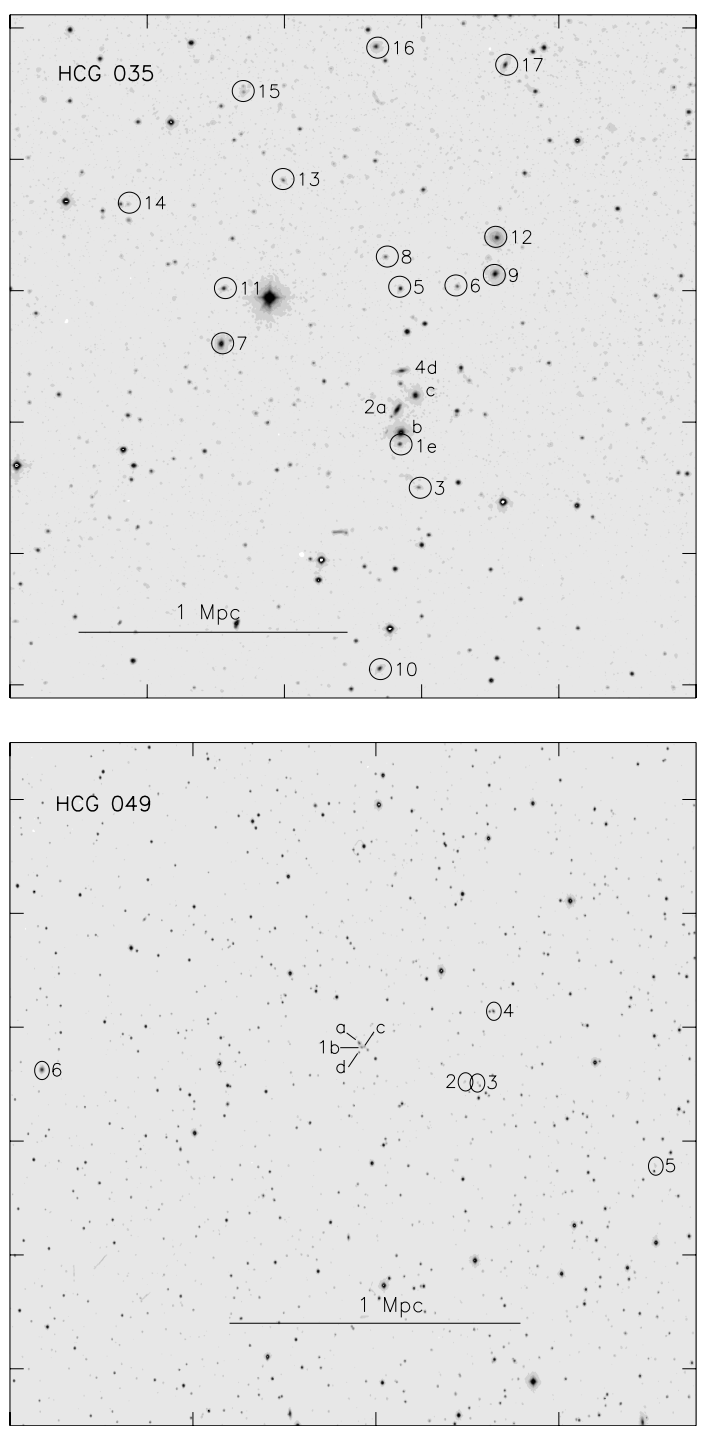

Fig. 1. continued. 
H. Tovmassian et al.: Hickson compact groups and their environment, Online Material p 3
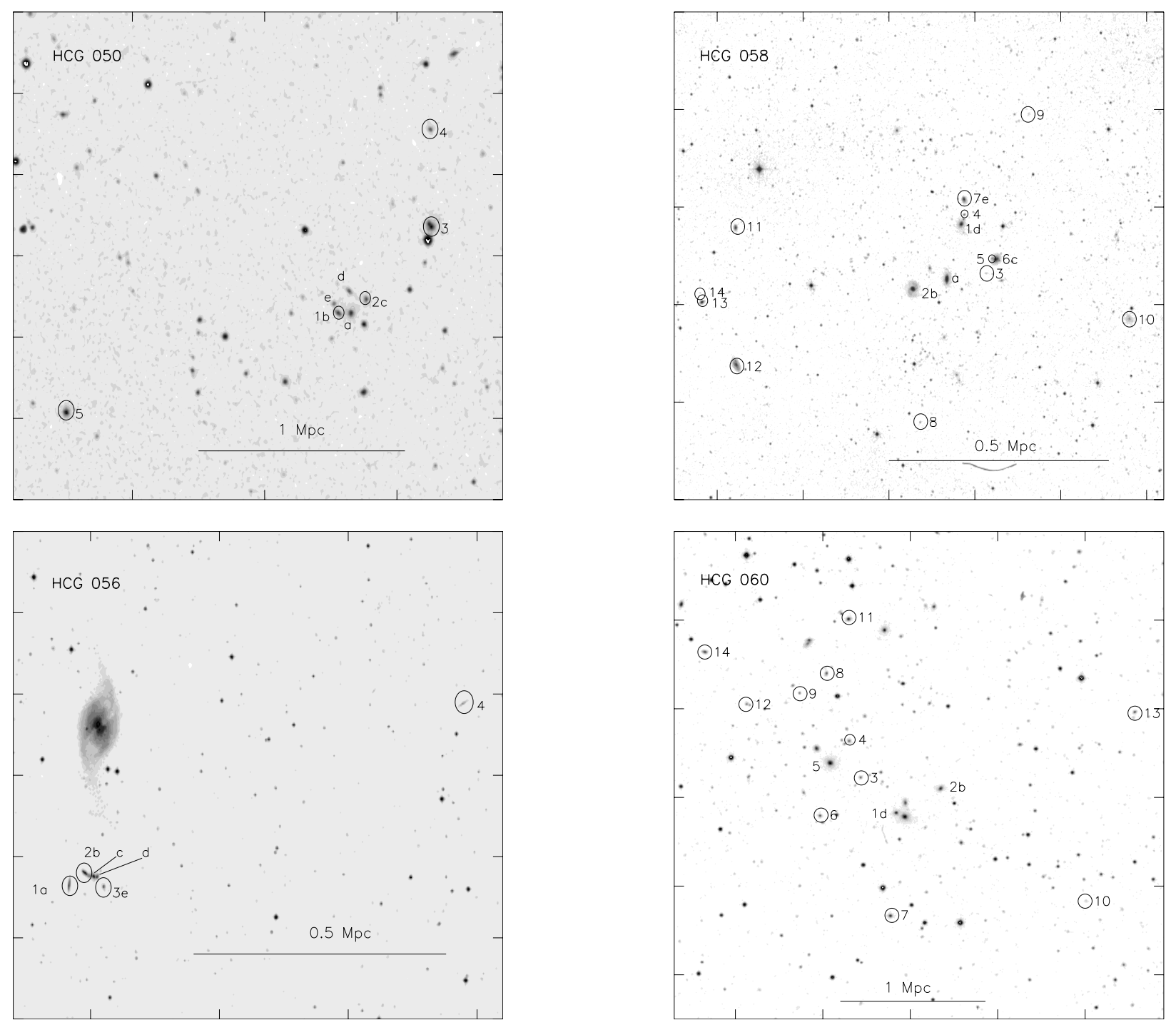

Fig. 1. continued.

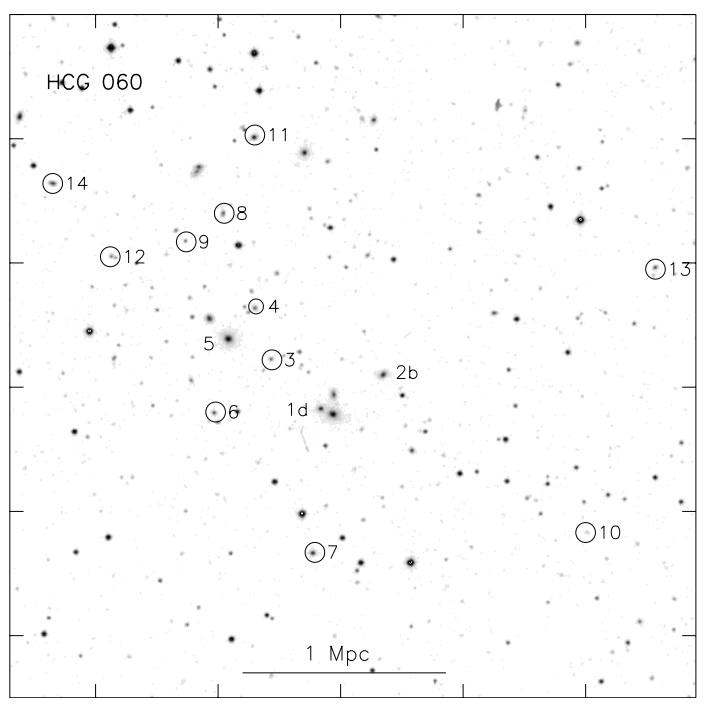

Fig. 1. continued. 
H. Tovmassian et al.: Hickson compact groups and their environment, Online Material p 4
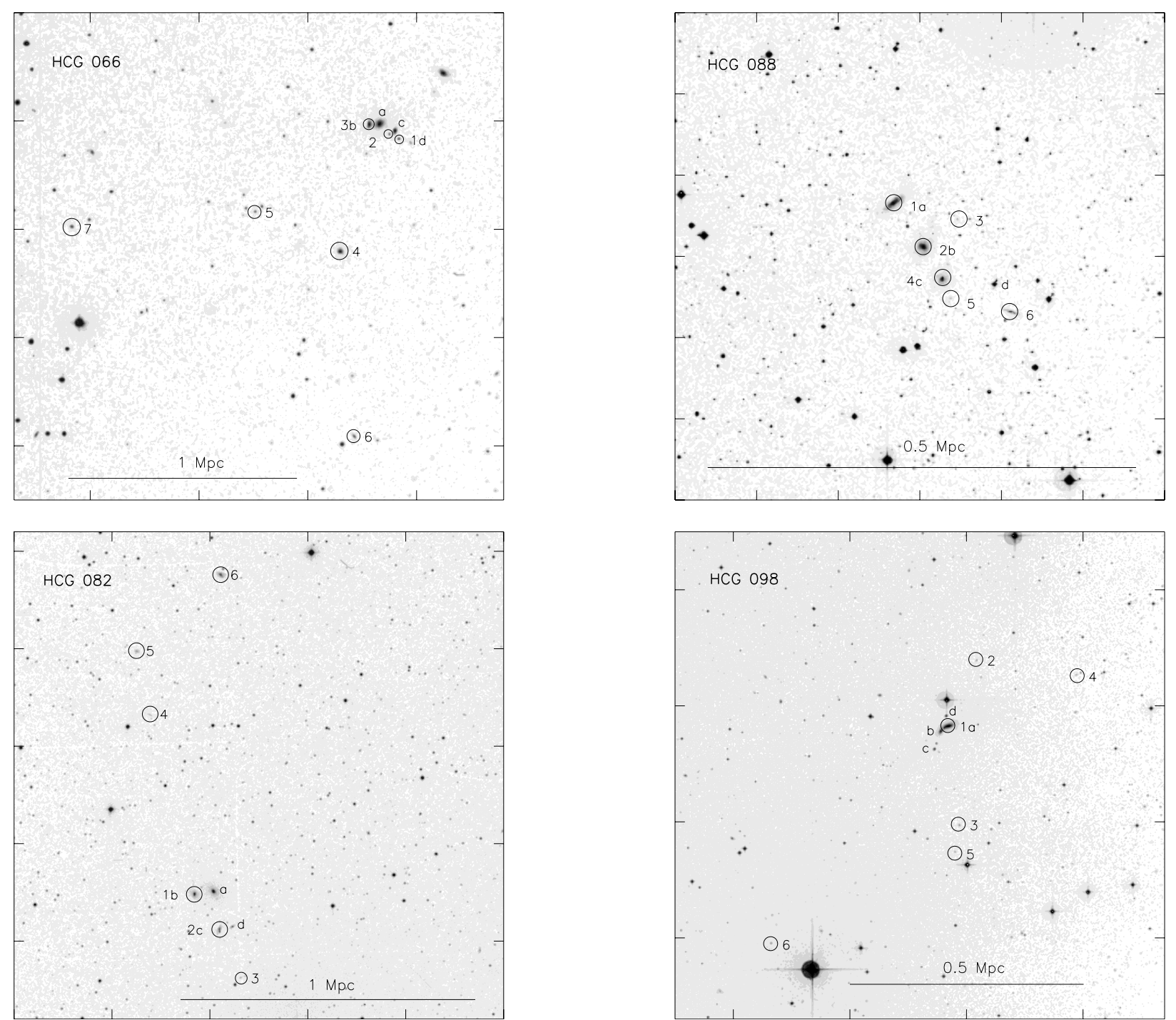

Fig. 1. continued.

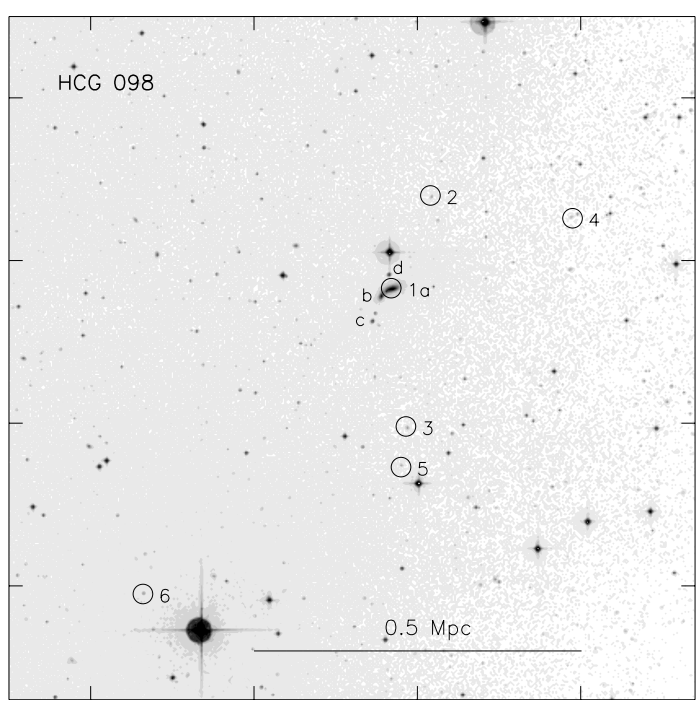

Fig. 1. continued.

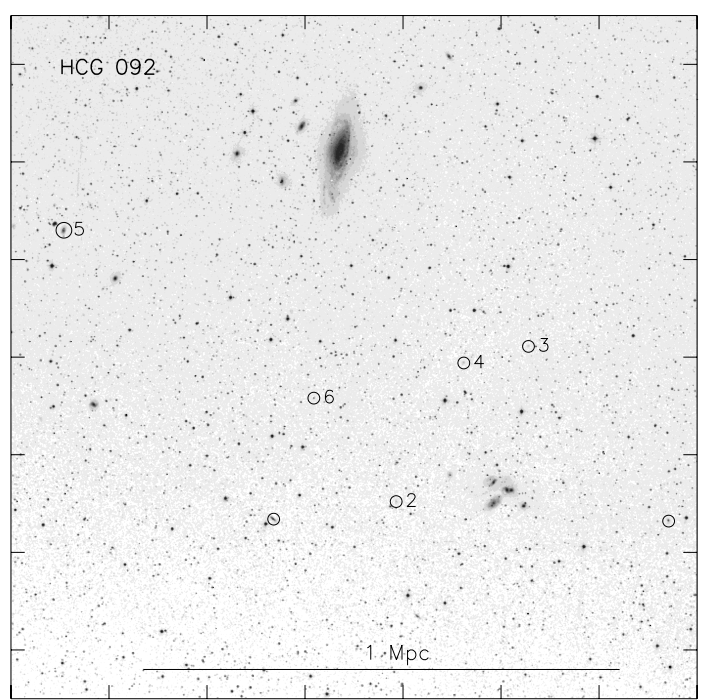

Fig. 1. continued. 